\title{
A non-abelian Hom-Leibniz tensor product and applications
}

\author{
J. M. Casas ${ }^{(1)}$, E. Khmaladze ${ }^{(2)}$ and N. Pacheco Rego ${ }^{(3)}$ \\ ${ }^{(1)}$ Dpto. Matemática Aplicada I, Univ. de Vigo, 36005 Pontevedra, Spain \\ e-mail address: jmcasas@uvigo.es \\ ${ }^{(2)}$ A. Razmadze Math. Inst. of I. Javakhishvili Tbilisi State University, \\ Tamarashvili Str. 6, 0177 Tbilisi, Georgia \\ e-mail address: e.khmal@gmail.com \\ (3) IPCA, Dpto. de Ciências, Campus do IPCA, Lugar do Aldão \\ 4750-810 Vila Frescainha, S. Martinho, Barcelos, Portugal \\ e-mail address: natarego@gmail.com
}

\begin{abstract}
The notion of non-abelian Hom-Leibniz tensor product is introduced and some properties are established. This tensor product is used in the description of the universal $(\alpha$-)central extensions of Hom-Leibniz algebras. We also give its application to the Hochschild homology of Hom-associative algebras.
\end{abstract}

A. M. S. Subject Class. (2010): 17A30, 17B55, 17B60, 18G35, 18G60

Key words: Hom-Leibniz algebra, non-abelian tensor product, universal $(\alpha)$-central extension, Hom-associative algebra, Hochschild homology.

\section{Introduction}

Since the invention of Hom-Lie algebras as the algebraic model of deformed Lie algebras coming from twisted discretizations of vectors fields by Hartwig, Larsson and Silvestrov in [10], many papers appeared dealing with Hom-type generalizations of various algebraic structures (see for instance 1, 2, 3, 6, 7, 14, 15, 16, 17, 18, 19] and related references given therein). In particular, Makhlouf and Silvestrov introduced the notion of Hom-Leibniz algebras in [14], which simultaneously is a non-commutative generalization of Hom-Lie algebras and Hom-type generalization of Leibniz algebras. In this generalized framework, it is natural to seek for possible extensions of results in the categories of Lie or Leibniz algebras to the categories of Hom-Lie or Hom-Leibniz algebras. 
Recently, in [4], we developed the non-abelian Hom-Lie tensor product, extending the non-abelian Lie tensor product by Ellis [8] from Lie to Hom-Lie algebras. It has applications in universal $(\alpha$-)central extensions of Hom-Lie algebras and cyclic homology of Hom-associative algebras.

In this paper we have chosen to work with Hom-Leibniz algebras. This work is a continuation and non-commutative generalization of the work already begun in [4]. Thus, we introduce a non-abelian Hom-Leibniz tensor product, extending the non-abelian Leibniz tensor product by Gnedbaye [9], which itself is the Leibniz algebra (non-commutative) version of the non-abelian Lie tensor product [8, 11]. Then we investigate properties of the non-abelian Hom-Leibniz tensor product (Section 3) and give its applications to universal $(\alpha$-)central extensions of Hom-Leibniz algebras (Section 4) and Hochschild homology of Hom-associative algebras (Section 5).

One observes that not all results can be transferred from Leibniz to HomLeibniz algebras. For example, results on universal central extensions of Leibniz algebras can not be extended directly to Hom-Leibniz algebras because the category of Hom-Leibniz algebras doesn't satisfy the so-called universal central extension condition [5], which means that the composition of central extensions is not central in general [3]. By this reason, the notion of $\alpha$-central extension of Hom-Leibniz algebras is introduced in [3], and classical results are divided between universal central and universal $\alpha$-central extensions of Hom-Leibniz algebras (see Theorem 4.31). Further, Hom-type version of Gnedbaye's result relating Hochschild and Milnor type Hochschild homology of associative algebras [9], doesn't hold for all Hom-associative algebras and requires an additional condition (see Theorem 5.7).

\section{Notations}

Throughout this paper we fix $\mathbb{K}$ as a ground field. Vector spaces are considered over $\mathbb{K}$ and linear maps are $\mathbb{K}$-linear maps. We write $\otimes$ for the tensor product $\otimes_{\mathbb{K}}$ over $\mathbb{K}$. For any vector space (resp. Hom-Leibniz algebra) $L$, a subspace (resp. a two-sided ideal) $L^{\prime}$ and $x \in L$ we write $\bar{x}$ to denote the coset $x+L^{\prime}$. We denote by Lie and Lb the categories of Lie and Leibniz algebras, respectively.

\section{Preliminaries on Hom-Leibniz algebras}

\section{$2.1 \quad$ Basic definitions}

In this section we review some terminology on Hom-Leibniz algebras and recall notions used in the paper. We also introduce notions of actions and semi-direct product of Hom-Leibniz algebras. 
Definition 2.1. 14] A Hom-Leibniz algebra is a triple $\left(L,[-,-], \alpha_{L}\right)$ consisting of a vector space $L$, a bilinear map $[-,-]: L \times L \rightarrow L$, called bracket operation, and a linear map $\alpha_{L}: L \rightarrow L$ satisfying:

$$
\left[\alpha_{L}(x),[y, z]\right]=\left[[x, y], \alpha_{L}(z)\right]-\left[[x, z], \alpha_{L}(y)\right] \quad \text { (Hom-Leibniz identity) }
$$

for all $x, y, z \in L$.

In the whole paper we only deal with (the so-called multiplicative [19]) HomLeibniz algebras $\left(L,[-,-], \alpha_{L}\right)$ such that $\alpha_{L}$ preserves the bracket operation, that is, $\alpha_{L}[x, y]=\left[\alpha_{L}(x), \alpha_{L}(y)\right]$, for all $x, y \in L$.

\section{Example 2.2.}

a) Taking $\alpha=$ id in Definition 2.1, we obtain the definition of a Leibniz algebra [12]. Hence any Leibniz algebra $L$ can be considered as a Hom-Leibniz algebra with $\alpha_{L}=$ id.

b) Any Leibniz algebra $L$ can be considered as a Hom-Leibniz algebra with $\alpha_{L}=0$. In fact, any vector space $L$ endowed with any bracket operation is a Hom-Leibniz algebra with $\alpha_{L}=0$.

c) Any Hom-Lie algebra [10] is a Hom-Leibniz algebra whose bracket operation satisfies the skew-symmetry condition.

d) Any Hom-dialgebra [18] $\left(D,-1, \vdash, \alpha_{D}\right)$ becomes a Hom-Leibniz algebra $(D$, $\left.[-,-], \alpha_{D}\right)$ with the bracket given by $[x, y]=x \dashv y-y \vdash x$, for all $x, y \in D$.

e) Let $(L,[-,-])$ be a Leibniz algebra and $\alpha_{L}: L \rightarrow L$ a Leibniz algebra endomorphism. Define $[-,-]_{\alpha}: L \otimes L \rightarrow L$ by $[x, y]_{\alpha}=[\alpha(x), \alpha(y)]$, for all $x, y \in L$. Then $\left(L,[-,-]_{\alpha}, \alpha_{L}\right)$ is a Hom-Leibniz algebra.

f) Any Hom-vector space $\left(V, \alpha_{V}\right)$, (i.e. $V$ is a vector space and $\alpha_{V}: V \rightarrow V$ is a linear map) together with the trivial bracket $[-,-]$ (i.e. $[x, y]=0$ for all $x, y \in V)$ is a Hom-Leibniz algebra, called abelian Hom-Leibniz algebra.

g) The two-dimensional $\mathbb{C}$-vector space $L$ with basis $\left\{a_{1}, a_{2}\right\}$, endowed with the bracket operation $\left[a_{2}, a_{2}\right]=a_{1}$ and zero elsewhere, and the endomorphism $\alpha_{L}$ given by the matrix $\left(\begin{array}{ll}1 & 1 \\ 0 & 1\end{array}\right)$ is a non-Hom-Lie Hom-Leibniz algebra.

In the sequel we shall use the shortened notation $\left(L, \alpha_{L}\right)$ for $\left(L,[-,-], \alpha_{L}\right)$.

Definition 2.3. A homomorphism of Hom-Leibniz algebras $f:\left(L, \alpha_{L}\right) \rightarrow\left(L^{\prime}, \alpha_{L^{\prime}}\right)$ is a linear map $f: L \rightarrow L^{\prime}$ such that

$$
\begin{aligned}
& f([x, y])=[f(x), f(y)], \\
& f \circ \alpha_{L}=\alpha_{L^{\prime}} \circ f,
\end{aligned}
$$

for all $x, y \in L$. 
Hom-Leibniz algebras and their homomorphisms form a category, which we denote by HomLb. Example 2.2 a), b) and c) say that there are full embedding functors

$$
I_{0}, I_{1}: \mathrm{Lb} \longrightarrow \text { HomLb }, \quad I_{0}(L)=(L, 0), I_{1}(L)=(L, \mathrm{id})
$$

and

$$
\text { inc : HomLie } \longrightarrow \text { HomLb, } \quad\left(L, \alpha_{L}\right) \mapsto\left(L, \alpha_{L}\right),
$$

where HomLie denotes the category of Hom-Lie algebras. Note that the restrictions of $I_{0}$ and $I_{1}$ to the category of Lie algebras are full embeddings

$$
I_{0}^{\prime}, I_{1}^{\prime}: \text { Lie } \longrightarrow \text { HomLie }, \quad I_{0}^{\prime}(L)=(L, 0), I_{1}^{\prime}(L)=(L, \text { id }) .
$$

Definition 2.4. A Hom-Leibniz subalgebra $\left(H, \alpha_{H}\right)$ of a Hom-Leibniz algebra $\left(L, \alpha_{L}\right)$ consists of a vector subspace $H$ of $L$, which is closed under the bracket, together with the linear map $\alpha_{H}: H \rightarrow H$ being the restriction of $\alpha_{L}$ on $H$. In such a case we may write $\alpha_{L \mid}$ for $\alpha_{H}$.

A Hom-Leibniz subalgebra $\left(H, \alpha_{H}\right)$ of $\left(L, \alpha_{L}\right)$ is said to be a two-sided Homideal if $[x, y],[y, x] \in H$, for all $x \in H, y \in L$.

If $\left(H, \alpha_{H}\right)$ is a two-sided Hom-ideal of $\left(L, \alpha_{L}\right)$, then the quotient vector space $L / H$ together with the endomorphism $\bar{\alpha}_{L}: L / H \rightarrow L / H$ induced by $\alpha_{L}$, naturally inherits a structure of Hom-Leibniz algebra, and it is called the quotient HomLeibniz algebra.

The commutator of two-sided Hom-ideals $\left(H, \alpha_{L \mid}\right)$ and $\left(K, \alpha_{L \mid}\right)$ of a HomLeibniz algebra $\left(L, \alpha_{L}\right)$, denoted by $\left([H, K], \alpha_{L \mid}\right)$, is the Hom-Leibniz subalgebra of $\left(L, \alpha_{L}\right)$ spanned by the brackets $[h, k]$ and $[k, h]$ for all $h \in H, k \in K$.

The following lemma can be readily checked.

Lemma 2.5. Let $\left(H, \alpha_{H}\right)$ and $\left(K, \alpha_{K}\right)$ be two-sided Hom-ideals of a Hom-Leibniz algebra $\left(L, \alpha_{L}\right)$. The following statements hold:

a) $\left(H \cap K, \alpha_{L \mid}\right)$ and $\left(H+K, \alpha_{L \mid}\right)$ are two-sided Hom-ideals of $\left(L, \alpha_{L}\right)$;

b) $[H, K] \subseteq H \cap K$;

c) If $\alpha_{L}$ is surjective, then $\left([H, K], \alpha_{L \mid}\right)$ is a two-sided Hom-ideal of $\left(L, \alpha_{L}\right)$;

d) $\left([H, K], \alpha_{L \mid}\right)$ is a two-sided Hom-ideal of $\left(H, \alpha_{H}\right)$ and $\left(K, \alpha_{K}\right)$. In particular, $\left([L, L], \alpha_{L \mid}\right)$ is a two-sided Hom-ideal of $\left(L, \alpha_{L}\right)$;

e) $\left(\alpha_{L}(L), \alpha_{L \mid}\right)$ is a Hom-Leibniz subalgebra of $\left(L, \alpha_{L}\right)$;

f) If $H, K \subseteq \alpha_{L}(L)$, then $\left([H, K], \alpha_{L \mid}\right)$ is a two-sided Hom-ideal of $\left(\alpha_{L}(L), \alpha_{L \mid}\right)$.

Definition 2.6. Let $\left(L, \alpha_{L}\right)$ be a Hom-Leibniz algebra. The subspace $Z(L)=$ $\{x \in L \mid[x, y]=0=[y, x]$, for all $y \in L\}$ of $L$ is said to be the center of $\left(L, \alpha_{L}\right)$. 
Note that if $\alpha_{L}: L \rightarrow L$ is a surjective homomorphism, then $\left(Z(L), \alpha_{L \mid}\right)$ is a two-sided Hom-ideal of $L$.

Corollary 2.7. Any Hom-Leibniz algebra $\left(L, \alpha_{L}\right)$ gives rise to a Hom-Lie algebra $\left(L_{\mathrm{Lie}}, \bar{\alpha}_{L}\right)$, which is obtained as the quotient of $L$ by the relation $[x, x]=0, x \in L$. Here $\bar{\alpha}_{L}$ is induced by $\alpha_{L}$. This defines a functor $(-)_{\text {Lie }}:$ HomLb $\longrightarrow$ HomLie. Moreover, the canonical epimorphism $\left(L, \alpha_{L}\right) \rightarrow\left(L_{\mathrm{Lie}}, \bar{\alpha}_{L}\right)$ is universal among all homomorphisms from $\left(L, \alpha_{L}\right)$ to a Hom-Lie algebra, implying that the functor $(-)_{\text {Lie }}$ is left adjoint to the inclusion functor inc : HomLie $\longrightarrow$ HomLb. This adjunction is a natural extension of the well-known adjunction $\mathrm{Lie} \underset{\text { inc }}{\stackrel{(-) \text { Lie }}{\longrightarrow}} \mathrm{Lb}$ between the categories of Lie and Leibniz algebras, in the sense that the following inner and outer diagrams

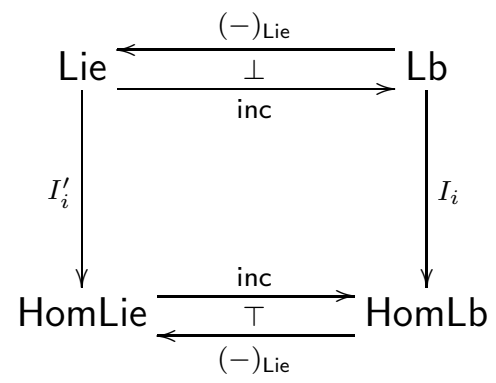

are commutative for $i=0,1$.

\subsection{Hom-Leibniz actions and semi-direct product}

Definition 2.8. Let $\left(L, \alpha_{L}\right)$ and $\left(M, \alpha_{M}\right)$ be Hom-Leibniz algebras. A HomLeibniz action of $\left(L, \alpha_{L}\right)$ on $\left(M, \alpha_{M}\right)$ is a couple of linear maps, $L \otimes M \rightarrow M$, $x \otimes m \mapsto{ }^{x} m$ and $M \otimes L \rightarrow M, m \otimes x \mapsto m^{x}$, satisfying the following identities:

a) $\alpha_{M}(m)^{[x, y]}=\left(m^{x}\right)^{\alpha_{L}(y)}-\left(m^{y}\right)^{\alpha_{L}(x)}$,

b) ${ }^{[x, y]} \alpha_{M}(m)=\left({ }^{x} m\right)^{\alpha_{L}(y)}-{ }^{\alpha_{L}(x)}\left(m^{y}\right)$,

c) ${ }^{\alpha_{L}(x)}\left({ }^{y} m\right)=-{ }^{\alpha_{L}(x)}\left(m^{y}\right)$,

d) ${ }^{\alpha_{L}(x)}\left[m, m^{\prime}\right]=\left[{ }^{x} m, \alpha_{M}\left(m^{\prime}\right)\right]-\left[{ }^{x} m^{\prime}, \alpha_{M}(m)\right]$,

e) $\left[m, m^{\prime}\right]^{\alpha_{L}(x)}=\left[m^{x}, \alpha_{M}\left(m^{\prime}\right)\right]+\left[\alpha_{M}(m), m^{\prime}{ }^{x}\right]$,

f) $\left[\alpha_{M}(m),{ }^{x} m^{\prime}\right]=-\left[\alpha_{M}(m), m^{\prime x}\right]$,

g) $\alpha_{M}\left({ }^{x} m\right)={ }^{\alpha_{L}(x)} \alpha_{M}(m)$,

h) $\alpha_{M}\left(m^{x}\right)=\alpha_{M}(m)^{\alpha_{L}(x)}$, 
for all $x, y \in L$ and $m, m^{\prime} \in M$.

The action is called trivial if ${ }^{x} m=0=m^{x}$, for all $x \in L, m \in M$.

\section{Example 2.9.}

a) A Hom-representation of a Hom-Leibniz algebra $\left(L, \alpha_{L}\right)$ is a Hom-vector space $\left(M, \alpha_{M}\right)$ equipped with two linear operations, $L \otimes M \rightarrow M, x \otimes m \mapsto$ ${ }^{x} m$ and $M \otimes L \rightarrow M, m \otimes x \mapsto m^{x}$, satisfying the axioms $\left.\left.\left.\left.a\right), b\right), c\right), g\right)$, h) of Definition 2.8. Therefore, representations over a Hom-Leibniz algebra $\left(L, \alpha_{L}\right)$ are abelian Leibniz algebras enriched with Hom-Leibniz actions of $\left(L, \alpha_{L}\right)$.

b) Any action of a Leibniz algebra $L$ on another Leibniz algebra $M$ (see e.g. [13]) gives a Hom-Leibniz action of $\left(L, \mathrm{id}_{L}\right)$ on $\left(M, \mathrm{id}_{M}\right)$.

c) Let $\left(K, \alpha_{K}\right)$ be a Hom-Leibniz subalgebra of a Hom-Leibniz algebra $\left(L, \alpha_{L}\right)$ and $\left(H, \alpha_{H}\right)$ be a two-sided Hom-ideal of $\left(L, \alpha_{L}\right)$. There exists a HomLeibniz action of $\left(K, \alpha_{K}\right)$ on $\left(H, \alpha_{H}\right)$ given by the bracket in $\left(L, \alpha_{L}\right)$.

d) Let $0 \rightarrow\left(M, \alpha_{M}\right) \stackrel{i}{\rightarrow}\left(K, \alpha_{K}\right) \stackrel{\pi}{\rightarrow}\left(L, \alpha_{L}\right) \rightarrow 0$ be a split short exact sequence of Hom-Leibniz algebras, $i$. e. there exists a homomorphism of Hom-Leibniz algebras $s:\left(L, \alpha_{L}\right) \rightarrow\left(K, \alpha_{K}\right)$ such that $\pi \circ s=\mathrm{id}_{L}$. Then there is a Hom-Leibniz action of $\left(L, \alpha_{L}\right)$ on $\left(M, \alpha_{M}\right)$ defined in the standard way: ${ }^{x} m=i^{-1}[s(x), i(m)]$ and $m^{x}=i^{-1}[i(m), s(x)]$ for all $x \in L, m \in M$.

Definition 2.10. Let $\left(M, \alpha_{M}\right)$ and $\left(L, \alpha_{L}\right)$ be Hom-Leibniz algebras together with a Hom-Leibniz action of $\left(L, \alpha_{L}\right)$ on $\left(M, \alpha_{M}\right)$. Their semi-direct product $\left(M \rtimes L, \alpha_{\rtimes}\right)$ is the Hom-Leibniz algebra with the underlying vector space $M \oplus L$, endomorphism $\alpha_{\rtimes}: M \rtimes L \rightarrow M \rtimes L$ given by $\alpha_{\rtimes}(m, l)=\left(\alpha_{M}(m), \alpha_{L}(l)\right)$ and bracket

$$
\left[\left(m_{1}, l_{1}\right),\left(m_{2}, l_{2}\right)\right]=\left(\left[m_{1}, m_{2}\right]+{ }^{\alpha_{L}\left(l_{1}\right)} m_{2}+m_{1}^{\alpha_{L}\left(l_{2}\right)},\left[l_{1}, l_{2}\right]\right) .
$$

Let $\left(M, \alpha_{M}\right)$ and $\left(L, \alpha_{L}\right)$ be Hom-Leibniz algebras with a Hom-Leibniz action of $\left(L, \alpha_{L}\right)$ on $\left(M, \alpha_{M}\right)$. Then we have the following short exact sequence of HomLeibniz algebras

$$
0 \rightarrow\left(M, \alpha_{M}\right) \stackrel{i}{\rightarrow}\left(M \rtimes L, \alpha_{\rtimes}\right) \stackrel{\pi}{\rightarrow}\left(L, \alpha_{L}\right) \rightarrow 0
$$

where $i: M \rightarrow M \rtimes L, i(m)=(m, 0)$, and $\pi: M \rtimes L \rightarrow L, \pi(m, x)=x$. Moreover, this sequence splits by the Hom-Leibniz homomorphism $L \rightarrow M \rtimes L$, $x \mapsto(0, x)$. 


\subsection{Homology of Hom-Leibniz algebras}

In this subsection we recall from [3] the construction of homology vector spaces of a Hom-Leibniz algebra with coefficients in a Hom-co-representation.

Definition 2.11. A Hom-co-representation of a Hom-Leibniz algebra $\left(L, \alpha_{L}\right)$ is a Hom-vector space $\left(M, \alpha_{M}\right)$ together with two linear maps $L \otimes M \rightarrow M, x \otimes m \mapsto$ ${ }^{x} m$ and $M \otimes L \rightarrow M, m \otimes x=m^{x}$, satisfying the following identities:

a) ${ }^{[x, y]} \alpha_{M}(m)={ }^{\alpha_{L}(x)}\left({ }^{y} m\right)-{ }^{\alpha_{L}(y)}\left({ }^{x} m\right)$,

b) $\alpha_{M}(m)^{[x, y]}=\left({ }^{y} m\right)^{\alpha_{L}(x)}-{ }^{\alpha_{L}(y)}\left(m^{x}\right)$,

c) $\left(m^{x}\right)^{\alpha_{L}(y)}=-{ }^{\alpha_{L}(y)}\left(m^{x}\right)$,

d) $\alpha_{M}\left({ }^{x} m\right)={ }^{\alpha_{L}(x)} \alpha_{M}(m)$,

e) $\alpha_{M}\left(m^{x}\right)=\alpha_{M}(m)^{\alpha_{L}(x)}$,

for any $x, y \in L$ and $m \in M$

\section{Example 2.12.}

a) Let $M$ be a co-representation of a Leibniz algebra $L$ [13], then $\left(M, \mathrm{id}_{M}\right)$ is a Hom-co-representation of the Hom-Leibniz algebra $\left(L, \mathrm{id}_{L}\right)$.

b) The underlying Hom-vector space of a Hom-Leibniz algebra $\left(L, \alpha_{L}\right)$ has a Hom-co-representation structure given by ${ }^{x} y=-[y, x]$ and $y^{x}=[y, x]$, $x, y \in L$.

Let $\left(L, \alpha_{L}\right)$ be a Hom-Leibniz algebra and $\left(M, \alpha_{M}\right)$ be a Hom-co-representation of $\left(L, \alpha_{L}\right)$. The homology $H L_{\star}^{\alpha}(L, M)$ of $\left(L, \alpha_{L}\right)$ with coefficients in $\left(M, \alpha_{M}\right)$ is defined to be the homology of the chain complex $\left(C L_{\star}^{\alpha}(L, M), d_{\star}\right)$, where

$$
C L_{n}^{\alpha}(L, M):=M \otimes L^{\otimes n}, \quad n \geq 0
$$

and $d_{n}: C L_{n}^{\alpha}(L, M) \rightarrow C L_{n-1}^{\alpha}(L, M), n \geq 1$, is the linear map given by

$$
\begin{aligned}
& d_{n}\left(m \otimes x_{1} \otimes \cdots \otimes x_{n}\right)=m^{x_{1}} \otimes \alpha_{L}\left(x_{2}\right) \otimes \cdots \otimes \alpha_{L}\left(x_{n}\right) \\
&+\sum_{i=2}^{n}(-1)^{i} \quad x_{i} m \otimes \alpha_{L}\left(x_{1}\right) \otimes \cdots \otimes \widehat{\alpha_{L}\left(x_{i}\right)} \otimes \cdots \otimes \alpha_{L}\left(x_{n}\right) \\
&+\sum_{1 \leq i<j \leq n}(-1)^{j+1} \alpha_{M}(m) \otimes \alpha_{L}\left(x_{1}\right) \otimes \cdots \otimes \alpha_{L}\left(x_{i-1}\right) \otimes\left[x_{i}, x_{j}\right] \otimes \alpha_{L}\left(x_{i+1}\right) \\
& \otimes \cdots \otimes \widehat{\alpha_{L}\left(x_{j}\right)} \otimes \cdots \otimes \alpha_{L}\left(x_{n}\right),
\end{aligned}
$$

where the notation $\widehat{\alpha_{L}\left(x_{i}\right)}$ indicates that the variable $\alpha_{L}\left(x_{i}\right)$ is omitted. Hence

$$
H L_{n}^{\alpha}(L, M):=H_{n}\left(C L_{\star}^{\alpha}(L, M), d_{\star}\right), \quad n \geq 0 .
$$


Direct calculations show that $H L_{0}^{\alpha}(L, M)=M / M^{L}$, where $M^{L}=\left\{m^{x} \mid m \in\right.$ $M, x \in L\}$, and if $\left(M, \alpha_{M}\right)$ is a trivial Hom-co-representation of $\left(L, \alpha_{L}\right)$, that is $m^{x}={ }^{x} m=0$, then $H L_{1}^{\alpha}(L, M)=(M \otimes L) /\left(\alpha_{M}(M) \otimes[L, L]\right)$. In particular, if $M=\mathbb{K}$ then $H L_{1}^{\alpha}(L, \mathbb{K})=L /[L, L]$. Later on we write $H L_{n}^{\alpha}(L)$ for $H L_{n}^{\alpha}(L, \mathbb{K})$ and it is called homology with trivial coefficients.

\section{Non-abelian Hom-Leibniz tensor product}

Let $\left(M, \alpha_{M}\right)$ and $\left(N, \alpha_{N}\right)$ be Hom-Leibniz algebras acting on each other. We denote by $M * N$ the vector space spanned by all symbols $m * n, n * m$ and subject to the following relations:

$$
\begin{aligned}
\lambda(m * n) & =(\lambda m) * n=m *(\lambda n), \\
\lambda(n * m) & =b(\lambda n) * m=n *(\lambda m), \\
\left(m+m^{\prime}\right) * n & =m * n+m^{\prime} * n, \\
m *\left(n+n^{\prime}\right) & =m * n+m * n^{\prime}, \\
\left(n+n^{\prime}\right) * m & =n * m+n^{\prime} * m, \\
n *\left(m+m^{\prime}\right) & =n * m+n * m^{\prime}, \\
\alpha_{M}(m) *\left[n, n^{\prime}\right] & =m^{n} * \alpha_{N}\left(n^{\prime}\right)-m^{n^{\prime}} * \alpha_{N}(n), \\
\alpha_{N}(n) *\left[m, m^{\prime}\right] & =n^{m} * \alpha_{M}\left(m^{\prime}\right)-n^{m^{\prime}} * \alpha_{M}(m), \\
{\left[m, m^{\prime}\right] * \alpha_{N}(n) } & ={ }^{m} n * \alpha_{M}\left(m^{\prime}\right)-\alpha_{M}(m) * n^{m^{\prime}}, \\
{\left[n, n^{\prime}\right] * \alpha_{M}(m) } & ={ }^{n} m * \alpha_{N}\left(n^{\prime}\right)-\alpha_{N}(n) * m^{n^{\prime}}, \\
\alpha_{M}(m) * m^{\prime} n & =-\alpha_{M}(m) * n^{m^{\prime}}, \\
\alpha_{N}(n) *{ }^{\prime} m & =-\alpha_{N}(n) * m^{n^{\prime}}, \\
m^{n} * m^{\prime} n^{\prime} & =m_{n} * m^{\prime n^{\prime}}, \\
m^{n} * n^{\prime m^{\prime}} & =m_{n} * n^{\prime} m^{\prime}, \\
n_{m} * m^{\prime} n^{\prime} & =n^{m} * m^{\prime n^{\prime}}, \\
n_{m} * n^{\prime m^{\prime}} & =n^{m} * n^{n^{\prime}} m^{\prime},
\end{aligned}
$$

for all $\lambda \in \mathbb{K}, m, m^{\prime} \in M, n, n^{\prime} \in N$.

We claim that $\left(M * N, \alpha_{M * N}\right)$ is a Hom-vector space, where $\alpha_{M * N}$ is the linear map induced by $\alpha_{M}$ and $\alpha_{N}$, i.e.

$$
\alpha_{M * N}(m * n)=\alpha_{M}(m) * \alpha_{N}(n), \quad \alpha_{M * N}(n * m)=\alpha_{N}(n) * \alpha_{M}(m) .
$$

Indeed, it can be checked readily that $\alpha_{*}$ preserves all the relations in (3.1).

To be able to introduce the non-abelian Hom-Leibniz tensor product, we need to assume that the actions are compatibly in the following sense.

Definition 3.1. Let $\left(M, \alpha_{M}\right)$ and $\left(N, \alpha_{N}\right)$ be Hom-Leibniz algebras with Hom- 
Leibniz actions on each other. The actions are said to be compatible if

$$
\begin{array}{ll}
{ }^{\left({ }^{m} n\right)} m^{\prime}=\left[m^{n}, m^{\prime}\right], & \left.{ }^{(n} m\right) n^{\prime}=b\left[n^{m}, n^{\prime}\right], \\
{ }^{\left(n^{m}\right)} m^{\prime}=\left[{ }^{n} m, m^{\prime}\right], & { }^{\left(m^{n}\right)} n^{\prime}=\left[{ }^{m} n, n^{\prime}\right], \\
m^{\left(m^{\prime} n\right)}=\left[m, m^{\prime} n\right], & n^{\left(n^{\prime} m\right)}=\left[n, n^{\prime}{ }^{m}\right], \\
m^{\left(n^{m^{\prime}}\right)}=\left[m,{ }^{n} m^{\prime}\right], & n^{\left(m^{n^{\prime}}\right)}=\left[n{ }^{m} n^{\prime}\right],
\end{array}
$$

for all $m, m^{\prime} \in M$ and $n, n^{\prime} \in N$.

Example 3.2. If $\left(H, \alpha_{H}\right)$ and $\left(H^{\prime}, \alpha_{H^{\prime}}\right)$ both are Hom-ideals of a Hom-Leibniz algebra $\left(L, \alpha_{L}\right)$, then the Hom-Leibniz actions of $\left(H, \alpha_{H}\right)$ and $\left(H^{\prime}, \alpha_{H^{\prime}}\right)$ on each other, considered in Example 2.9 c), are compatible.

Now we have the following property:

Proposition 3.3. Let $\left(M, \alpha_{M}\right)$ and $\left(N, \alpha_{N}\right)$ be Hom-Leibniz algebras acting compatibly on each other, then the Hom-vector space $\left(M * N, \alpha_{M * N}\right)$ endowed with the following bracket operation

$$
\begin{aligned}
& {\left[m * n, m^{\prime} * n^{\prime}\right]=m^{n} * m^{m^{\prime}} n^{\prime},} \\
& {\left[m * n, n^{\prime} * m^{\prime}\right]=m^{n} * n^{\prime m^{\prime}},} \\
& {\left[n * m, m^{\prime} * n^{\prime}\right]={ }^{n} m * m^{m^{\prime}} n^{\prime},} \\
& {\left[n * m, n^{\prime} * m^{\prime}\right]={ }^{n} m * n^{\prime m^{\prime}},}
\end{aligned}
$$

is a Hom-Leibniz algebra.

Proof. Routine calculations show that the bracket given by (3.3) is compatible with the defining relations in (3.1) of $M * N$ and can be extended from generators to any elements. The verification of the Hom-Leibniz identity (2.1) is straightforward by using compatibility conditions in (3.2). Finally, it follows directly by definition of $\alpha_{M * N}$ that it preserves the bracket given by (3.3).

Definition 3.4. The above Hom-Leibniz algebra structure on $\left(M * N, \alpha_{M * N}\right)$ is called the non-abelian Hom-Leibniz tensor product of the Hom-Leibniz algebras $\left(M, \alpha_{M}\right)$ and $\left(N, \alpha_{N}\right)$.

Remark 3.5. If $\alpha_{M}=\mathrm{id}_{M}$ and $\alpha_{N}=\mathrm{id}_{N}$, then $M * N$ coincides with the nonabelian tensor product of Leibniz algebras introduced in [9].

Remark 3.6. Let $\left(M, \alpha_{M}\right)$ and $\left(N, \alpha_{N}\right)$ be Hom-Lie algebras. One can readily check that the following assertions hold:

a) Any Hom-Lie action of $\left(M, \alpha_{M}\right)$ on $\left(N, \alpha_{N}\right), M \otimes N \rightarrow N, m \otimes n \mapsto{ }^{m} n$ (see [4. Definition 1.7] for the definition), gives a Hom-Leibniz action of $\left(M, \alpha_{M}\right)$ on $\left(N, \alpha_{N}\right)$ by letting $n^{m}=-{ }^{m} n$ for all $m \in M$ and $n \in N$. 
b) For compatible Hom-Lie actions (see [4, Definition 2.1]) of $\left(M, \alpha_{M}\right)$ and $\left(N, \alpha_{N}\right)$ on each other, the induced Hom-Leibniz actions are also compatible.

c) If $\left(M, \alpha_{M}\right)$ and $\left(N, \alpha_{N}\right)$ act compatibly on each other, then there is an epimorphism of Hom-Leibniz algebras $\left(M * N, \alpha_{M * N}\right) \rightarrow\left(M \star N, \alpha_{M \star N}\right)$ defined on generators by $m * n \mapsto m \star n$ and $n * m \mapsto-m \star n$, where $\star$ denotes the non-abelian Hom-Lie tensor product (see [4, Definition 2.4]).

Sometimes the non-abelian Hom-Leibniz tensor product can be described as the tensor product of vector spaces. In particular, we have the following:

Proposition 3.7. If the Hom-Leibniz algebras $\left(M, \alpha_{M}\right)$ and $\left(N, \alpha_{N}\right)$ act trivially on each other and both $\alpha_{M}, \alpha_{N}$ are epimorphisms, then there is an isomorphism of abelian Hom-Leibniz algebras

$$
\left(M * N, \alpha_{M * N}\right) \cong\left(\left(M^{a b} \otimes N^{a b}\right) \oplus\left(N^{a b} \otimes M^{a b}\right), \alpha_{\oplus}\right),
$$

where $M^{a b}=M /[M, M], N^{a b}=N /[N, N]$ and $\alpha_{\oplus}$ denotes the linear self-map of $\left(M^{a b} \otimes N^{a b}\right) \oplus\left(N^{a b} \otimes M^{a b}\right)$ induced by $\alpha_{M}$ and $\alpha_{N}$.

Proof. Since the actions are trivial, then relations (3.3) enables us to see that $\left(M * N, \alpha_{M * N}\right)$ is an abelian Hom-Leibniz algebra.

Since $\alpha_{M}$ and $\alpha_{N}$ are epimorphisms, the defining relations (3.1) of the nonabelian tensor product say precisely that the vector space $M * N$ is the quotient of $(M \otimes N) \oplus(N \otimes M)$ by the relations

$$
m *\left[n, n^{\prime}\right]=\left[m, m^{\prime}\right] * n=\left[n, n^{\prime}\right] * m=n *\left[m, m^{\prime}\right]=0
$$

for all $m, m^{\prime} \in M, n, n^{\prime} \in N$. The later is isomorphic to $\left(M^{a b} \otimes N^{a b}\right) \oplus\left(N^{a b} \otimes M^{a b}\right)$ and this isomorphism commutes with the endomorphisms $\alpha_{\oplus}$ and $\alpha_{M * N}$.

The non-abelian Hom-Leibniz tensor product is functorial in the following sense: if $f:\left(M, \alpha_{M}\right) \rightarrow\left(M^{\prime}, \alpha_{M^{\prime}}\right)$ and $g:\left(N, \alpha_{N}\right) \rightarrow\left(N^{\prime}, \alpha_{N^{\prime}}\right)$ are homomorphisms of Hom-Leibniz algebras together with compatible actions of $\left(M, \alpha_{M}\right)$ (resp. $\left.\left(M^{\prime}, \alpha_{M^{\prime}}\right)\right)$ and $\left(N, \alpha_{N}\right)$ (resp. $\left.\left(N^{\prime}, \alpha_{N^{\prime}}\right)\right)$ on each other such that $f, g$ preserve these actions, that is

$$
\begin{aligned}
& f\left({ }^{n} m\right)=g(n) f(m), \quad f\left(m^{n}\right)=f(m)^{g(n)}, \\
& g\left({ }^{m} n\right)={ }^{f(m)} g(n), \quad g\left(n^{m}\right)=g(n)^{f(m)},
\end{aligned}
$$

for all $m \in M, n \in N$, then we have a homomorphism of Hom-Leibniz algebras

$$
f * g:\left(M * N, \alpha_{M * N}\right) \rightarrow\left(M^{\prime} * N^{\prime}, \alpha_{M^{\prime} * N^{\prime}}\right)
$$

defined by $(f * g)(m * n)=f(m) * g(n),(f * g)(n * m)=g(n) * f(m)$. 
Proposition 3.8. Let $0 \rightarrow\left(M_{1}, \alpha_{M_{1}}\right) \stackrel{f}{\rightarrow}\left(M_{2}, \alpha_{M_{2}}\right) \stackrel{g}{\rightarrow}\left(M_{3}, \alpha_{M_{3}}\right) \rightarrow 0$ be a short exact sequence of Hom-Leibniz algebras. Let $\left(N, \alpha_{N}\right)$ be a Hom-Leibniz algebra together with compatible Hom-Leibniz actions of $\left(N, \alpha_{N}\right)$ and $\left(M_{i}, \alpha_{M_{i}}\right)$ $(i=1,2,3)$ on each other and $f, g$ preserve these actions. Then there is an exact sequence of Hom-Leibniz algebras

$$
\left(M_{1} * N, \alpha_{M_{1} * N}\right) \stackrel{f * \mathrm{id}_{N}}{\longrightarrow}\left(M_{2} * N, \alpha_{M_{2} * N}\right) \stackrel{g * \mathrm{id}_{N}}{\longrightarrow}\left(M_{3} * N, \alpha_{M_{3} * N}\right) \longrightarrow 0 .
$$

Proof. Clearly $g * \mathrm{id}_{N}$ is an epimorphism and $\operatorname{Im}\left(f * \mathrm{id}_{N}\right) \subseteq \operatorname{Ker}\left(g * \mathrm{id}_{N}\right)$. Since $\operatorname{Im}\left(f * \mathrm{id}_{N}\right)$ is generated by all elements of the form $f\left(m_{1}\right) * n, n * f\left(m_{1}\right)$, with $m_{1} \in M_{1}, n \in N$, it is a two-sided Hom-ideal in $\left(M_{2} * N, \alpha_{M_{2} * N}\right)$ because of the following equalities:

$$
\begin{aligned}
& {\left[f\left(m_{1}\right) * n, m_{2} * n^{\prime}\right]=f\left(m_{1}\right)^{n} *^{m_{2}} n^{\prime}=f\left(m_{1}{ }^{n}\right) * m_{2} n^{\prime} \in \operatorname{Im}\left(f * \mathrm{id}_{N}\right),} \\
& {\left[f\left(m_{1}\right) * n, n^{\prime} * m_{2}\right]=f\left(m_{1}\right)^{n} * n^{\prime} m_{2}=f\left(m_{1}^{n}\right) * n^{\prime} m_{2} \in \operatorname{Im}\left(f * \mathrm{id}_{N}\right) \text {, }} \\
& {\left[n * f\left(m_{1}\right), m_{2} * n^{\prime}\right]={ }^{n} f\left(m_{1}\right) *{ }^{m_{2}} n^{\prime}=f\left({ }^{n} m_{1}\right) *{ }^{m_{2}} n^{\prime} \in \operatorname{Im}\left(f * \mathrm{id}_{N}\right) \text {, }} \\
& {\left[n * f\left(m_{1}\right), n^{\prime} * m_{2}\right]={ }^{n} f\left(m_{1}\right) * n^{\prime} m_{2}=f\left({ }^{n} m_{1}\right) * n^{\prime} m_{2} \in \operatorname{Im}\left(f * \mathrm{id}_{N}\right) \text {, }} \\
& {\left[m_{2} * n^{\prime}, f\left(m_{1}\right) * n\right]=m_{2}{ }^{n^{\prime}} *{ }^{f\left(m_{1}\right)} n=m_{2}{ }^{n^{\prime}} * f\left(m_{1} n\right) \in \operatorname{Im}\left(f * \mathrm{id}_{N}\right) \text {, }} \\
& {\left[m_{2} * n^{\prime}, n * f\left(m_{1}\right)\right]=m_{2}{ }^{n^{\prime}} * n^{f\left(m_{1}\right)}=m_{2}^{n^{\prime}} * f\left(n^{m_{1}}\right) \in \operatorname{Im}\left(f * \mathrm{id}_{N}\right) \text {, }} \\
& {\left[n^{\prime} * m_{2}, f\left(m_{1}\right) * n\right]={ }^{\prime} m_{2} *{ }^{f\left(m_{1}\right)} n=n^{\prime} m_{2} * f\left({ }^{m_{1}} n\right) \in \operatorname{Im}\left(f * \mathrm{id}_{N}\right) \text {, }} \\
& {\left[n^{\prime} * m_{2}, n * f\left(m_{1}\right)\right]=n^{\prime} m_{2} * n^{f\left(m_{1}\right)}=n^{\prime} m_{2} * f\left(n^{m_{1}}\right) \in \operatorname{Im}\left(f * \mathrm{id}_{N}\right) \text {, }} \\
& \alpha_{M_{2} * N}\left(f\left(m_{1}\right) * n\right)=f\left(\alpha_{M_{2}}\left(m_{1}\right)\right) * \alpha_{N}(n) \in \operatorname{Im}\left(f * \mathrm{id}_{N}\right), \\
& \alpha_{M_{2} * N}\left(n * f\left(m_{1}\right)\right)=f\left(\alpha_{N}(n) * \alpha_{M_{2}}\left(m_{1}\right)\right) \in \operatorname{Im}\left(f * \mathrm{id}_{N}\right) \text {, }
\end{aligned}
$$

for any $m_{1} \in M_{1}, m_{2} \in M_{2}, n, n^{\prime} \in N$. Thus, $g * \mathrm{id}_{N}$ induces a homomorphism of Hom-Leibniz algebras

$$
\left(\left(M_{2} * N\right) / \operatorname{Im}\left(f * \mathrm{id}_{N}\right), \bar{\alpha}_{M_{2} * N}\right) \longrightarrow\left(M_{3} * N, \alpha_{M_{3} * N}\right),
$$

given on generators by $\overline{m_{2} * n} \mapsto g\left(m_{2}\right) * n$ and $\overline{n * m_{2}} \mapsto n * g\left(m_{2}\right)$, which is an isomorphism with the inverse map

$$
\left(M_{3} * N, \alpha_{M_{3} * N}\right) \longrightarrow\left(\left(M_{2} * N\right) / \operatorname{Im}\left(f * \mathrm{id}_{N}\right), \bar{\alpha}_{M_{2} * N}\right)
$$

defined by $m_{3} * n \mapsto \overline{m_{2} * n}$ and $n * m_{3}=\overline{n * m_{2}}$, where $m_{2} \in M_{2}$ such that $g\left(m_{2}\right)=m_{3}$. Then the required exactness follows.

Proposition 3.9. If $\left(M, \alpha_{M}\right)$ is a two-sided Hom-ideal of a Hom-Leibniz algebra $\left(L, \alpha_{L}\right)$, then there is an exact sequence of Hom-Leibniz algebras

$$
\left((M * L) \rtimes(L * M), \alpha_{\rtimes}\right) \stackrel{\sigma}{\longrightarrow}\left(L * L, \alpha_{L * L}\right) \stackrel{\tau}{\longrightarrow}\left(L / M * L / M, \alpha_{L / M * L / M}\right) \longrightarrow 0 .
$$

where the Hom-Leibniz action of $L * M$ on $M * L$ is naturally given by the bracket in $L * M$. 
Proof. First we note that $\tau$ is the functorial homomorphism induced by projection $\left(L, \alpha_{L}\right) \rightarrow\left(L / M, \alpha_{L / M}\right)$ and clearly it is an epimorphism.

Let $\sigma^{\prime}:\left(M * L, \alpha_{M * L}\right) \rightarrow\left(L * L, \alpha_{L * L}\right), \sigma^{\prime \prime}:\left(L * M, \alpha_{L * M}\right) \rightarrow\left(L * L, \alpha_{L * L}\right)$ be the functorial homomorphisms induced by the inclusion $\left(M, \alpha_{M}\right) \hookrightarrow\left(L, \alpha_{L}\right)$ and the identity map $\left(L, \alpha_{L}\right) \rightarrow\left(L, \alpha_{L}\right)$. Then $\sigma:\left((M * L) \rtimes(L * M), \alpha_{\rtimes}\right) \rightarrow$ $\left(L * L, \alpha_{L * L}\right)$, is defined by $\sigma(x, y)=\sigma^{\prime}(x)+\alpha_{L * L} \circ \sigma^{\prime \prime}(y)$ for all $x \in M * L$ and $y \in L * M$, i. e.

$$
\begin{aligned}
& \sigma\left(\left(m_{1} * l_{1}\right),\left(l_{2} * m_{2}\right)\right)=m_{1} * l_{1}+\alpha_{L}\left(l_{2}\right) * \alpha_{M}\left(m_{2}\right), \\
& \sigma\left(\left(l_{1} * m_{1}\right),\left(l_{2} * m_{2}\right)\right)=l_{1} * m_{1}+\alpha_{L}\left(l_{2}\right) * \alpha_{M}\left(m_{2}\right), \\
& \sigma\left(\left(m_{1} * l_{1}\right),\left(m_{2} * l_{2}\right)\right)=m_{1} * l_{1}+\alpha_{M}\left(m_{2}\right) * \alpha_{L}\left(l_{2}\right), \\
& \sigma\left(\left(l_{1} * m_{1}\right),\left(m_{2} * l_{2}\right)\right)=l_{1} * m_{1}+\alpha_{M}\left(m_{2}\right) * \alpha_{L}\left(l_{2}\right),
\end{aligned}
$$

for all $m_{1}, m_{2} \in M$ and $l_{1}, l_{2} \in L$.

The exactness can be checked in the same way as in the proof of Proposition 3.8 and we omit it.

Proposition 3.10. Let $\left(M, \alpha_{M}\right)$ and $\left(N, \alpha_{N}\right)$ be Hom-Leibniz algebras with compatible actions on each other.

a) There are homomorphisms of Hom-Leibniz algebras

$$
\begin{array}{lll}
\psi_{1}:\left(M * N, \alpha_{M * N}\right) \rightarrow\left(M, \alpha_{M}\right), & \psi_{1}(m * n)=m^{n}, & \psi_{1}(n * m)={ }^{n} m, \\
\psi_{2}:\left(M * N, \alpha_{M * N}\right) \rightarrow\left(N, \alpha_{N}\right), & \psi_{2}(m * n)={ }^{m} n, & \psi_{2}(n * m)=n^{m} .
\end{array}
$$

b) There is a Hom-Leibniz action of $\left(M, \alpha_{M}\right)$ (resp. $\left.\left(N, \alpha_{N}\right)\right)$ on the nonabelian tensor product $\left(M * N, \alpha_{M * N}\right)$ given, for all $m, m^{\prime} \in M, n, n^{\prime} \in N$, by

$$
\begin{aligned}
m^{\prime}(m * n) & =\left[m^{\prime}, m\right] * \alpha_{N}(n)-m^{m^{\prime}} n * \alpha_{M}(m), \\
m^{\prime}(n * m) & ={ }^{\prime} n * \alpha_{M}(m)-\left[m^{\prime}, m\right] * \alpha_{N}(n), \\
(m * n)^{m^{\prime}} & =\left[m, m^{\prime}\right] * \alpha_{N}(n)+\alpha_{M}(m) * n^{m^{\prime}}, \\
(n * m)^{m^{\prime}} & =n^{m^{\prime}} * \alpha_{M}(m)+\alpha_{N}(n) *\left[m, m^{\prime}\right], \\
\left(\text { resp. }{ }^{n^{\prime}}(m * n)\right. & ={ }^{\prime} m * \alpha_{N}(n)-\left[n^{\prime}, n\right] * \alpha_{M}(m), \\
n^{\prime}(n * m) & =\left[n^{\prime}, n\right] * \alpha_{M}(m)-{ }^{n} m * \alpha_{N}\left(n^{\prime}\right), \\
(m * n)^{n^{\prime}} & =m^{n^{\prime}} * \alpha_{N}(n)+\alpha_{M}(m) *\left[n, n^{\prime}\right], \\
(n * m)^{n^{\prime}} & \left.=\left[n, n^{\prime}\right] * \alpha_{M}(m)+\alpha_{N}(n) * m^{n^{\prime}}\right) .
\end{aligned}
$$

d) $\operatorname{Ker}\left(\psi_{1}\right)$ and $\operatorname{Ker}\left(\psi_{2}\right)$ both are contained in the center of $\left(M * N, \alpha_{M * N}\right)$.

e) The induced Hom-Leibniz action of $\operatorname{Im}\left(\psi_{1}\right)$ (resp. $\left.\operatorname{Im}\left(\psi_{2}\right)\right)$ on $\operatorname{Ker}\left(\psi_{1}\right)$ (resp. $\left.\operatorname{Ker}\left(\psi_{2}\right)\right)$ is trivial.

f) $\psi_{1}$ and $\psi_{2}$ satisfy the following properties for all $m, m^{\prime} \in M, n, n^{\prime} \in N$ : 


$$
\begin{aligned}
& \text { i) } \psi_{1}\left(m^{\prime}(m * n)\right)=\left[\alpha_{M}\left(m^{\prime}\right), \psi_{1}(m * n)\right], \\
& \text { ii) } \psi_{1}\left((m * n)^{m^{\prime}}\right)=\left[\psi_{1}(m * n), \alpha_{M}\left(m^{\prime}\right)\right], \\
& \text { iii) } \psi_{1}\left(m^{\prime}(n * m)\right)=\left[\alpha_{M}\left(m^{\prime}\right), \psi_{1}(n * m)\right], \\
& \text { iv) } \psi_{1}\left((n * m)^{m^{\prime}}\right)=\left[\psi_{1}(n * m), \alpha_{M}\left(m^{\prime}\right)\right], \\
& \text { v) } \psi_{2}\left(n^{\prime}(m * n)\right)=\left[\alpha_{N}\left(n^{\prime}\right), \psi_{2}(m * n)\right], \\
& \text { vi) } \psi_{2}\left((m * n)^{n^{\prime}}\right)=\left[\psi_{2}(m * n), \alpha_{N}\left(n^{\prime}\right)\right], \\
& \text { vii) } \psi_{2}\left(n^{\prime}(n * m)\right)=\left[\alpha_{N}\left(n^{\prime}\right), \psi_{2}(n * m)\right], \\
& \text { viii) } \psi_{2}\left((n * m)^{n^{\prime}}\right)=\left[\psi_{2}(n * m), \alpha_{N}\left(n^{\prime}\right)\right], \\
& \text { ix) } \psi_{1}(m * n)\left(m^{\prime} * n^{\prime}\right)=\left[\alpha_{M * N}(m * n), m^{\prime} * n^{\prime}\right]=\psi_{2}(m * n)\left(m^{\prime} * n^{\prime}\right), \\
& \text { x) } \psi_{1}(m * n)\left(n^{\prime} * m^{\prime}\right)=\left[\alpha_{M * N}(m * n), n^{\prime} * m^{\prime}\right]=\psi_{2}(m * n)\left(n^{\prime} * m^{\prime}\right), \\
& \text { xi) } \psi_{1}(n * m)\left(m^{\prime} * n^{\prime}\right)=\left[\alpha_{M * N}(n * m), m^{\prime} * n^{\prime}\right]=\psi_{2}(n * m)\left(m^{\prime} * n^{\prime}\right), \\
& \text { xii) } \psi_{1}(n * m)\left(n^{\prime} * m^{\prime}\right)=\left[\alpha_{M * N}(n * m), n^{\prime} * m^{\prime}\right]=\psi_{2}(n * m)\left(n^{\prime} * m^{\prime}\right), \\
& \text { xiii) }\left(m^{\prime} * n^{\prime}\right)^{\psi_{1}(m * n)}=\left[m^{\prime} * n^{\prime}, \alpha_{M * N}(m * n)\right]=\left(m^{\prime} * n^{\prime}\right)^{\psi_{2}(m * n)}, \\
& \text { xiv) }\left(n^{\prime} * m^{\prime}\right)^{\psi_{1}(m * n)}=\left[n^{\prime} * m^{\prime}, \alpha_{M * N}(m * n)\right]=\left(n^{\prime} * m^{\prime}\right)^{\psi_{2}(m * n)}, \\
& \text { xv) }\left(m^{\prime} * n^{\prime}\right)^{\psi_{1}(n * m)}=\left[m^{\prime} * n^{\prime}, \alpha_{M * N}(n * m)\right]=\left(m^{\prime} * n^{\prime}\right)^{\psi_{2}(n * m)}, \\
& \text { xvi) }\left(n^{\prime} * m^{\prime}\right)^{\psi_{1}(n * m)}=\left[n^{\prime} * m^{\prime}, \alpha_{M * N}(n * m)\right]=\left(n^{\prime} * m^{\prime}\right)^{\psi_{2}(n * m)} .
\end{aligned}
$$

Proof. The proof requires routine combinations of equations in Definitions 2.8 and 3.1 with the defining relations (3.1) and (3.3) of the non-abelian Hom-Leibniz tensor product.

\section{Remark 3.11.}

a) If $\alpha_{M}=\mathrm{id}_{M}$ and $\alpha_{N}=\mathrm{id}_{N}$ then the statement $f$ ) of Lemma 3.10 says that both $\psi_{1}$ and $\psi_{2}$ are crossed modules of Leibniz algebras (see [9]).

b) If $M=N$ and the action of $M$ on itself is given by the bracket in $M$, then $\psi_{1}=\psi_{2}$ and they are defined on generators by $m * m^{\prime} \mapsto\left[m, m^{\prime}\right]$. In such a case we write $\psi_{M}$ for $\psi_{1}$.

\section{Application in universal $(\alpha-)$ central exten- sions of Hom-Leibniz algebras}

In this section we complement by new results the investigation of universal central extensions of Hom-Leibniz algebras done in [3]. We also describe universal $(\alpha)$ central extensions via non-abelian Hom-Leibniz tensor product. 
Definition 4.1. An epimorphism of Hom-Leibniz algebras $\left(K, \alpha_{K}\right) \stackrel{\pi}{\rightarrow}\left(L, \alpha_{L}\right)$, with $\operatorname{Ker}(\pi)=\left(M, \alpha_{M}\right)$, is called a central (resp. $\alpha$-central) extension of $\left(L, \alpha_{L}\right)$ if $[M, K]=0=[K, M]$, i.e. $M \subseteq Z(K)$ (resp. $\left[\alpha_{M}(M), K\right]=0=\left[K, \alpha_{M}(M)\right]$, i.e. $\left.\alpha_{M}(M) \subseteq Z(K)\right)$.

Such a central extension is called universal central (resp. $\alpha$-central) extension if, for every central (resp. $\alpha$-central) extension $\left(K^{\prime}, \alpha_{K^{\prime}}\right) \stackrel{\pi}{\rightarrow}\left(L, \alpha_{L}\right)$ of $\left(L, \alpha_{L}\right)$ there exists one and only one homomorphism of Hom-Leibniz algebras $h:\left(K, \alpha_{K}\right) \rightarrow\left(K^{\prime}, \alpha_{K^{\prime}}\right)$ such that $\pi^{\prime} \circ h=\pi$.

Remark 4.2. Obviously every central extension is an $\alpha$-central extension and these notions coincide when $\alpha_{M}=\mathrm{id}_{M}$. On the other hand, every universal $\alpha$ central extension is a universal central extension and these notions coincide when $\alpha_{M}=\mathrm{id}_{M}$. Let us also observe that if a universal $(\alpha$-) central extension exists then it is unique up to isomorphism.

The category HomLb is an example of a semi-abelian category which does not satisfy universal central extension condition in the sense of [5], that is, the composition of central extensions of Hom-Leibniz algebras is not central in general, but it is $\alpha$-central extension (see Theorem 4.3 (a) below). This fact does not allow complete generalization of classical results to Hom-Leibniz algebras and the well-known properties of universal central extensions are divided between universal central and universal $\alpha$-central extensions of Hom-Leibniz algebras. In particular, the assertions in Theorem 4.3 below are proved in [3].

To state the following theorem we need to recall that a Hom-Lie algebra $\left(L, \alpha_{L}\right)$ is said to be perfect if $L=[L, L]$.

\section{Theorem 4.3.}

a) Let $\left(K, \alpha_{K}\right) \stackrel{\pi}{\rightarrow}\left(L, \alpha_{L}\right)$ and $\left(F, \alpha_{F}\right) \stackrel{\rho}{\rightarrow}\left(K, \alpha_{K}\right)$ be central extensions of Hom-Leibniz algebras and $\left(K, \alpha_{K}\right)$ be a perfect Hom-Leibniz algebra. Then the composition extension $\left(F, \alpha_{F}\right) \stackrel{\pi \circ \rho}{\rightarrow}\left(L, \alpha_{L}\right)$ is an $\alpha$-central extension.

b) Let $\left(K, \alpha_{K}\right) \stackrel{\pi}{\rightarrow}\left(L, \alpha_{L}\right)$ and $\left(K^{\prime}, \alpha_{K^{\prime}}\right) \stackrel{\pi^{\prime}}{\rightarrow}\left(L, \alpha_{L}\right)$ be two central extensions of a Hom-Leibniz algebra $\left(L, \alpha_{L}\right)$. If $\left(K, \alpha_{K}\right)$ is perfect, then there exists at most one homomorphism of Hom-Leibniz algebras $f:\left(K, \alpha_{K}\right) \rightarrow\left(K^{\prime}, \alpha_{K^{\prime}}\right)$ such that $\pi^{\prime} \circ f=\pi$.

c) Let $\left(K, \alpha_{K}\right) \stackrel{\pi}{\rightarrow}\left(L, \alpha_{L}\right)$ be a central extension and $\left(K^{\prime}, \alpha_{K^{\prime}}\right) \stackrel{\pi^{\prime}}{\rightarrow}\left(L, \alpha_{L}\right)$ be an $\alpha$-central extension of a Hom-Leibniz algebra $\left(L, \alpha_{L}\right)$. If $\left(K, \alpha_{K}\right)$ is $\alpha$-perfect, then there exists at most one homomorphism of Hom-Leibniz algebras $f:\left(K, \alpha_{K}\right) \rightarrow\left(K^{\prime}, \alpha_{K^{\prime}}\right)$ such that $\pi^{\prime} \circ f=\pi$.

d) If $\left(K, \alpha_{K}\right) \stackrel{\pi}{\rightarrow}\left(L, \alpha_{L}\right)$ is a universal $\alpha$-central extension, then $\left(K, \alpha_{K}\right)$ is a perfect Hom-Leibniz algebra and every central extension of $\left(K, \alpha_{K}\right)$ splits. 
e) If $\left(K, \alpha_{K}\right)$ is a perfect Hom-Leibniz algebra and every central extension of $\left(K, \alpha_{K}\right)$ splits, then any central extension $\left(K, \alpha_{K}\right) \stackrel{\pi}{\rightarrow}\left(L, \alpha_{L}\right)$ is a universal central extension.

f) A Hom-Leibniz algebra $\left(L, \alpha_{L}\right)$ admits a universal central extension if and only if $\left(L, \alpha_{L}\right)$ is perfect. Furthermore, the kernel of the universal central extension is canonically isomorphic to the second homology $H L_{2}^{\alpha}(L)$.

g) An $\alpha$-perfect Hom-Leibniz algebra admits a universal $\alpha$-central extension.

h) If $\left(K, \alpha_{K}\right) \stackrel{\pi}{\rightarrow}\left(L, \alpha_{L}\right)$ is a universal $\alpha$-central extension, then $H L_{1}^{\alpha}(K)=$ $H L_{2}^{\alpha}(K)=0$.

i) If $H L_{1}^{\alpha}(K)=H L_{2}^{\alpha}(K)=0$, then any central extension $\left(K, \alpha_{K}\right) \stackrel{\pi}{\rightarrow}\left(L, \alpha_{L}\right)$ is a universal central extension.

Given a Hom-Leibniz algebra $\left(L, \alpha_{L}\right)$ we have the epimorphism of HomLeibniz algebras

$$
\psi_{L}:\left(L * L, \alpha_{L * L}\right) \rightarrow\left([L, L], \alpha_{L}\right), \quad \psi_{L}\left(l * l^{\prime}\right)=\left[l, l^{\prime}\right],
$$

(cf. Remark $3.11 \mathrm{~b})$ ) which is a central extension of $\left([L, L], \alpha_{L \mid}\right)$ because of the equalities (3.3). Moreover, we have

Theorem 4.4. If $\left(L, \alpha_{L}\right)$ is a perfect Hom-Leibniz algebra, then the central extension $\left(L * L, \alpha_{L * L}\right) \stackrel{\psi_{L}}{\rightarrow}\left(L, \alpha_{L}\right)$ is the universal central extension of $\left(L, \alpha_{L}\right)$.

Proof. Let $\left(C, \alpha_{C}\right) \stackrel{\phi}{\rightarrow}\left(L, \alpha_{L}\right)$ be a central extension of $\left(L, \alpha_{L}\right)$. Since $\operatorname{Ker}(\phi) \subseteq$ $Z(C)$, we get a well-defined homomorphism of Hom-Leibniz algebras $f:(L * L$, $\left.\alpha_{L * L}\right) \rightarrow\left(C, \alpha_{C}\right)$ given on generators by $f\left(l * l^{\prime}\right)=\left[c_{l}, c_{l^{\prime}}\right]$, where $c_{l}$ and $c_{l^{\prime}}$ are any elements in $\phi^{-1}(l)$ and $\phi^{-1}\left(l^{\prime}\right)$, respectively. Obviously $\phi \circ f=\psi_{L}$ and $f \circ \alpha_{L * L}=\alpha_{C} \circ f$, having in mind that $\alpha_{C}\left(c_{l}\right) \in \phi^{-1}\left(\alpha_{L}(l)\right)$ for all $l \in L$. Since $L$ is perfect, then by equalities (3.3), so is $L * L$. Hence the homomorphism $f$ is unique by Theorem $4.3 \mathrm{~b}$ ).

Remark 4.5. If the Hom-Leibniz algebra $\left(L, \alpha_{L}\right)$ is perfect, by Theorem 4.3 e) we have $H L_{2}^{\alpha}(L) \approx \operatorname{Ker}\left(L * L \stackrel{\psi_{L}}{\rightarrow} L\right)$.

Theorem 4.6. Let $\left(M, \alpha_{M}\right)$ be a two-sided Hom-ideal of a perfect Hom-Leibniz algebra $\left(L, \alpha_{L}\right)$. Then there is an exact sequence of vector spaces

$$
\operatorname{Ker}\left(L * M \stackrel{\psi_{2}}{\longrightarrow} L\right) \rightarrow H L_{2}^{\alpha}(L) \rightarrow H L_{2}^{\alpha}(L / M) \rightarrow M /[L, M] \rightarrow 0 .
$$


Proof. By Proposition 3.9 there is a commutative diagram of Hom-Leibniz algebras with exact rows

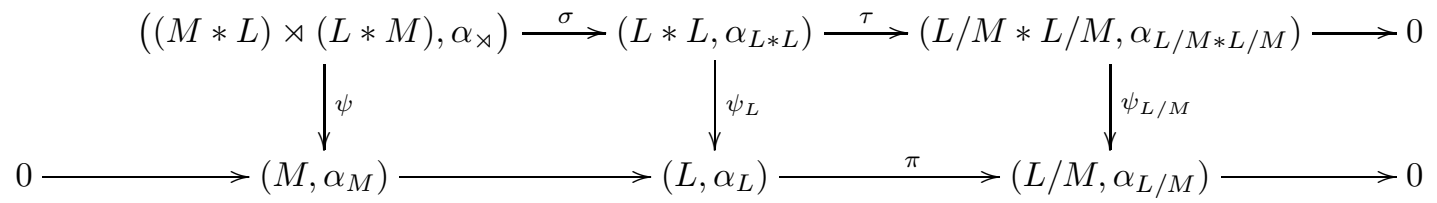

where

$$
\begin{aligned}
& \psi\left(\left(m_{1} * l_{1}\right),\left(l_{2} * m_{2}\right)\right)=\left[m_{1}, l_{1}\right]+\left[\alpha_{L}\left(l_{2}\right), \alpha_{M}\left(m_{2}\right)\right], \\
& \psi\left(\left(l_{1} * m_{1}\right),\left(l_{2} * m_{2}\right)\right)=\left[l_{1}, m_{1}\right]+\left[\alpha_{L}\left(l_{2}\right), \alpha_{M}\left(m_{2}\right)\right], \\
& \psi\left(\left(m_{1} * l_{1}\right),\left(m_{2} * l_{2}\right)\right)=\left[m_{1}, l_{1}\right]+\left[\alpha_{M}\left(m_{2}\right), \alpha_{L}\left(l_{2}\right)\right], \\
& \psi\left(\left(l_{1} * m_{1}\right),\left(m_{2} * l_{2}\right)\right)=\left[l_{1}, m_{1}\right]+\left[\alpha_{M}\left(m_{2}\right), \alpha_{L}\left(l_{2}\right)\right],
\end{aligned}
$$

and

$$
\begin{aligned}
\psi_{L}\left(l_{1} * l_{2}\right) & =\frac{\left[l_{1}, l_{2}\right]}{\left[l_{1}, l_{2}\right]}, \\
\psi_{L / M}\left(\overline{l_{1}} * \overline{l_{2}}\right) & =
\end{aligned}
$$

for all $m_{1}, m_{2} \in M, l_{1}, l_{2} \in L$. Then, forgetting the Hom-Leibniz algebra structures, by using the Snake Lemma for the same diagram of vector spaces, the assertion follows from Remark 4.5 and the fact that there is a surjective map $\operatorname{Ker}(\psi) \rightarrow \operatorname{Ker}\left(\psi_{2}\right)$.

Now suppose $\left(L, \alpha_{L}\right)$ is a perfect Hom-Lie algebra. By 4, Theorem 3.4 and Remark 3.5] we know that its universal central extension in the category Hom-Lie is

$$
\left(L \star L, \alpha_{L \star L}\right) \stackrel{u_{L}}{\rightarrow}\left(L, \alpha_{L}\right),
$$

and $\operatorname{Ker}\left(u_{L}\right) \approx H_{2}^{\alpha}(L)$, where $\star$ denotes the non-abelian Hom-Lie tensor product and $H_{2}^{\alpha}(L)$ is the second homology with trivial coefficients of the Hom-Lie algebra $\left(L, \alpha_{L}\right)$. Furthermore, since any perfect Hom-Lie algebra is a perfect Hom-Leibniz algebra as well, by Theorem $4.3 f),\left(L, \alpha_{L}\right)$ admits universal central extension in the category HomLb, which is described via the non-abelian Hom-Leibniz tensor product in Theorem 4.4 and Remark 4.5. Then, by the universal property of $(L *$ $\left.L, \alpha_{L * L}\right) \stackrel{\psi_{L}}{\rightarrow}\left(L, \alpha_{L}\right)$ and considering (4.1) as a central extension of Hom-Leibniz algebras, we get a homomorphism of Hom-Leibniz algebras $\xi:\left(L * L, \alpha_{L * L}\right) \rightarrow(L \star$ $\left.L, \alpha_{L \star L}\right), \xi\left(l * l^{\prime}\right)=l \star l^{\prime}$, such that $\psi_{L}=u_{L} \circ \xi$. Note that $\xi$ is the epimorphism described in Remark $3.6 \mathrm{c}$ ). Now the proof of the following proposition is obvious.

Proposition 4.7. Let $\left(L, \alpha_{L}\right)$ be a perfect Hom-Lie algebra, then the non-abelian Hom-Lie and Hom-Leibniz tensor squares are isomorphic, $\left(L \star L, \alpha_{L \star L}\right) \approx(L * L$, $\left.\alpha_{L * L}\right)$, if and only if the second Hom-Lie and Hom-Leibniz homologies with trivial coefficients are isomorphic, $H_{2}^{\alpha}(L) \approx H L_{2}^{\alpha}(L)$.

Now we deal with the universal $\alpha$-central extension of an $\alpha$-perfect HomLeibniz algebra. We need the following notion. 
Definition 4.8. [3] A Hom-Leibniz algebra $\left(L, \alpha_{L}\right)$ is said to be $\alpha$-perfect if

$$
L=\left[\alpha_{L}(L), \alpha_{L}(L)\right]
$$

\section{Remark 4.9.}

a) When $\alpha_{L}=$ id the notions of perfect and $\alpha$-perfect Hom-Leibniz algebras are the same.

b) Any $\alpha$-perfect Hom-Lie algebra is an $\alpha$-perfect Hom-Leibniz algebra.

c) Any $\alpha$-perfect Hom-Leibniz algebra is perfect. Nevertheless the converse is not true in general. An example of this is given in [4, Remark 3.8 b)].

d) If $\left(L, \alpha_{L}\right)$ is $\alpha$-perfect, then $L=\alpha_{L}(L)$, i.e. $\alpha_{L}$ is surjective.

It is shown in [3, Theorem 5.5 (b)] that an $\alpha$-perfect Hom-Leibniz algebra admits a universal $\alpha$-central extension. Now we give its description via the nonabelian Hom-Leibniz tensor product.

Theorem 4.10. Let $\left(L, \alpha_{L}\right)$ be an $\alpha$-perfect Hom-Leibniz algebra. Then the epimorphism $\psi:\left(\alpha_{L}(L) * \alpha_{L}(L), \alpha_{\alpha_{L}(L) * \alpha_{L}(L)}\right) \rightarrow\left(L, \alpha_{L}\right), \psi\left(\alpha_{L}(l) * \alpha_{L}\left(l^{\prime}\right)\right)=$ $\left[\alpha_{L}(l), \alpha_{L}\left(l^{\prime}\right)\right]$, is its universal $\alpha$-central extension.

Proof. Obviously $\psi$ is a central extension. For every $\alpha$-central extension $\left(C, \alpha_{C}\right) \stackrel{\phi}{\rightarrow}$ $\left(L, \alpha_{L}\right)$ there is a homomorphism $f:\left(\alpha_{L}(L) * \alpha_{L}(L), \alpha_{\alpha_{L}(L) * \alpha_{L}(L)}\right) \rightarrow\left(C, \alpha_{C}\right)$ given on generators by $f\left(\alpha_{L}(l) * \alpha_{L}\left(l^{\prime}\right)\right)=\left[\alpha_{C}\left(c_{l}\right), \alpha_{C}\left(c_{l^{\prime}}\right)\right]$, where $c_{l}$ and $c_{l^{\prime}}$ are any elements in $\phi^{-1}(l)$ and $\phi^{-1}\left(l^{\prime}\right)$, respectively. $f$ is well-defined since $\alpha_{C}(\operatorname{Ker}(\phi)) \subseteq Z(C)$. Obviously $\phi \circ f=\psi$ and $f \circ \alpha_{\alpha_{L}(L) * \alpha_{L}(L)}=\alpha_{C} \circ f$, having in mind that $\alpha_{C}\left(c_{l}\right) \in \phi^{-1}\left(\alpha_{L}(l)\right)$ and $\alpha_{C}^{2}\left(c_{l}\right) \in \phi^{-1}\left(\alpha_{L}^{2}(l)\right)$ for all $l \in L$. Since $L$ is $\alpha$-perfect, then by equality (3.3), so is $\alpha_{L}(L) * \alpha_{L}(L)$. Hence the homomorphism $f$ is unique by Theorem $4.3 \mathrm{c}$ ).

An alternative construction of the universal $\alpha$-central extension $\left(\mathfrak{u c e}_{\alpha}^{\mathrm{Lb}}(L), \bar{\alpha}\right)$ of an $\alpha$-perfect Hom-Leibniz algebra $\left(L, \alpha_{L}\right)$ is given in [3, Theorem $\left.5.5(\mathrm{~b})\right]$. Let us recall that $\mathfrak{u c e}_{\alpha}^{\mathrm{Lb}}(L)$ is the quotient of $\alpha_{L}(L) \otimes \alpha_{L}(L)$ by the vector subspace spanned by all elements $-\left[x_{1}, x_{2}\right] \otimes \alpha_{L}\left(x_{3}\right)+\left[x_{1}, x_{3}\right] \otimes \alpha_{L}\left(x_{2}\right)+\alpha_{L}\left(x_{1}\right) \otimes\left[x_{2}, x_{3}\right]$, where $x_{1}, x_{2}, x_{3} \in L$, the bracket of $\mathfrak{u c e}_{\alpha}^{\mathrm{Lb}}(L)$ is given on generators by

$$
\left[\alpha_{L}\left(x_{1}\right) \otimes \alpha_{L}\left(x_{2}\right), \alpha_{L}\left(y_{1}\right) \otimes \alpha_{L}\left(y_{2}\right)\right]=\left[\alpha_{L}\left(x_{1}\right), \alpha_{L}\left(x_{2}\right)\right] \otimes\left[\alpha_{L}\left(y_{1}\right), \alpha_{L}\left(y_{2}\right)\right],
$$

$x_{1}, x_{2}, y_{1}, y_{2} \in L$, and $\bar{\alpha}: \mathfrak{u c e}_{\alpha}^{\mathrm{Lb}}(L) \rightarrow \mathfrak{u c e}_{\alpha}^{\mathrm{Lb}}(L)$ is induced by $\alpha_{L}$.

Since the universal $\alpha$-central extension of an $\alpha$-perfect Hom-Leibniz algebra $\left(L, \alpha_{L}\right)$ is unique up to isomorphisms, then there is an isomorphism of HomLeibniz algebras

$$
\left(\alpha(L) * \alpha(L), \alpha_{\alpha(L) * \alpha(L)}\right) \approx\left(\mathfrak{u} \mathfrak{e r}_{\alpha}^{\mathrm{Lb}}(L), \bar{\alpha}\right) .
$$


Now suppose that $\left(L, \alpha_{L}\right)$ is an $\alpha$-perfect Hom-Lie algebra. By [3, Theorem 5.5 (a)] it admits a universal $\alpha$-central extension in the category HomLie of Hom-Lie algebras, which is described as the non-abelian Hom-Lie tensor product $\left(\alpha(L) \star \alpha(L), \alpha_{\alpha(L) \star \alpha(L)}\right)$ in [4, Theorem 3.11]. $\left(L, \alpha_{L}\right)$ admits a universal $\alpha$-central extension in the category HomLb described in Theorem 4.10 via nonabelian Hom-Leibniz tensor product. Then [3, Proposition 5.6] provides the following relationship between the non-abelian Hom-Lie and Hom-Leibniz tensor products:

$$
\left(\alpha(L) \star \alpha(L), \alpha_{\alpha(L) \star \alpha(L)}\right) \approx\left((\alpha(L) * \alpha(L))_{\text {Lie }}, \bar{\alpha}_{\alpha(L) * \alpha(L)}\right) .
$$

Moreover, $\left(\alpha(L) \star \alpha(L), \alpha_{\alpha(L) \star \alpha(L)}\right)$ is an $\alpha$-perfect Hom-Lie algebra and its universal $\alpha$-central extension in the category HomLb of Hom-Leibniz algebras is isomorphic to $\left(\alpha(L) * \alpha(L), \alpha_{\alpha(L) * \alpha(L)}\right)$.

\section{Application in Hochschild homology of Hom- associative algebras}

Definition 5.1. A (multiplicative) Hom-associative algebra $\left(A, \alpha_{A}\right)$ is a vector space $A$ together with linear maps $\alpha_{A}: A \rightarrow A$ and $A \otimes A \rightarrow A, a \otimes b \mapsto a b$, such that

$$
\begin{aligned}
& \alpha_{A}(a)(b c)=(a b) \alpha_{A}(c), \\
& \alpha_{A}(a b)=\alpha_{A}(a) \alpha_{A}(b)
\end{aligned}
$$

for all $a, b, c \in A$.

Any Hom-associative algebra $\left(A, \alpha_{A}\right)$ can be viewed as a Hom-Leibniz (in fact Hom-Lie) algebra with the bracket defined by $[a, b]:=a b-b a, a, b \in A$ (see Example 5.2 in [19]).

Given a Hom-associative algebra $\left(A, \alpha_{A}\right)$, we denote $\mathbb{L}^{\alpha}(A):=(A \otimes A) / \operatorname{Im}\left(b_{3}\right)$, where $b_{3}: A \otimes A \otimes A \rightarrow A \otimes A, b_{3}(a \otimes b \otimes c)=a b \otimes \alpha_{A}(c)-\alpha_{A}(a) \otimes b c+c a \otimes \alpha_{A}(b)$, is the boundary map for the Hochschild complex of the Hom-associative algebra $\left(A, \alpha_{A}\right)$ constructed in [19]. Then we have the following short exact sequence of vector spaces

$$
0 \longrightarrow H H_{1}^{\alpha}(A) \stackrel{i}{\longrightarrow} \mathbb{L}^{\alpha}(A) \stackrel{\phi}{\longrightarrow}[A, A] \longrightarrow 0,
$$

where $H H_{1}^{\alpha}(A)$ denotes the first Hochschild homology of $\left(A, \alpha_{A}\right)$ [19], $[A, A]$ is the subspace of $A$ spanned by the elements $a b-b a$ and $\phi(a \otimes b)=a b-b a$, $a, b \in A$. Here $a \otimes b$ stands for the equivalence class of $a \otimes b$.

Suppose $\bar{\alpha}_{A}: \mathbb{L}^{\alpha}(A) \rightarrow \mathbb{L}^{\alpha}(A)$ is the linear map induced by $\alpha_{A}$, i.e. $\bar{\alpha}_{A}(a \otimes b)=$ $\alpha_{A}(a) \otimes \alpha_{A}(b)$. Then there is a Hom-Leibniz algebra structure on $\left(\mathbb{L}^{\alpha}(A), \bar{\alpha}_{A}\right)$ given by the bracket

$$
\left[a \otimes b, a^{\prime} \otimes b^{\prime}\right]:=(a b-b a) \otimes\left(a^{\prime} b^{\prime}-b^{\prime} a^{\prime}\right)=[a, b] \otimes\left[a^{\prime}, b^{\prime}\right],
$$


for all $a, b, a^{\prime}, b^{\prime} \in A$. Indeed, it is immediate that

$$
[a, b] \bigotimes \alpha_{A}(c)-\alpha_{A}(a) \otimes[b, c]+[c, a] \otimes \alpha_{A}(b)=0,
$$

then the Hom-Leibniz identity (2.1) easily follows.

Remark 5.2. $\left(\mathbb{L}^{\alpha}(A), \bar{\alpha}_{A}\right)$ is the quotient of the non-abelian Hom-Leibniz tensor product $\left(A * A, \alpha_{A * A}\right)$ by the two-sided Hom-ideal generated by the elements of the form $a b * \alpha_{A}(c)-\alpha_{A}(a) * b c+c a * \alpha_{A}(b)$, for all $a, b, c \in A$.

Definition 5.3. [4] We say that a Hom-associative algebra $\left(A, \alpha_{A}\right)$ satisfies the $\alpha$-identity condition if

$$
\left[A, \operatorname{lm}\left(\alpha_{A}-\mathrm{id}_{A}\right)\right]=0,
$$

where $\left[A, \operatorname{Im}\left(\alpha_{A}-\mathrm{id}_{A}\right)\right]=0$ is the subspace of $A$ spanned by all elements $a b-b a$, with $a \in A$ and $b \in \operatorname{Im}\left(\alpha_{A}-\mathrm{id}_{A}\right)$.

Examples of Hom-associative algebras satisfying the $\alpha$-identity condition can be found in [4].

Proposition 5.4. Let $\left(A, \alpha_{A}\right)$ be a Hom-associative algebra.

a) There is a Hom-Leibniz action of $\left(A, \alpha_{A}\right)$ on $\left(\mathbb{L}^{\alpha}(A), \bar{\alpha}_{A}\right)$ given, for all $a^{\prime} \in A, a \bigotimes b \in \mathbb{L}^{\alpha}(A)$, by

$$
\begin{aligned}
a^{\prime}(a \otimes b) & =\left[a^{\prime}, a\right] \otimes \alpha_{A}(b)-\left[a^{\prime}, b\right] \otimes \alpha_{A}(a), \\
(a \bigotimes b)^{a^{\prime}} & =\left[a, a^{\prime}\right] \otimes \alpha_{A}(b)+\alpha_{A}(a) \otimes\left[b, a^{\prime}\right],
\end{aligned}
$$

and a Hom-Leibniz action of $\left(\mathbb{L}^{\alpha}(A), \bar{\alpha}_{A}\right)$ on $\left(A, \alpha_{A}\right)$ given by

$$
\begin{aligned}
{ }^{(a \Downarrow b)} a^{\prime} & =\left[[a, b], a^{\prime}\right], \\
a^{(a \unrhd b)} & =\left[a^{\prime},[a, b]\right] .
\end{aligned}
$$

Moreover, these actions are compatible if $\left(A, \alpha_{A}\right)$ satisfies the $\alpha$-identity condition (5.3).

b) There is a short exact sequence of Hom-Leibniz algebras

$$
0 \rightarrow\left(H H_{1}^{\alpha}(A), \bar{\alpha}_{A \mid}\right) \stackrel{i}{\rightarrow}\left(\mathbb{L}^{\alpha}(A), \bar{\alpha}_{A}\right) \stackrel{\phi}{\rightarrow}\left([A, A], \alpha_{A \mid}\right) \rightarrow 0
$$

where $\left(H H_{1}^{\alpha}(A), \alpha_{A \mid}\right)$ is considered as an abelian Hom-Leibniz algebra, $\bar{\alpha}_{A \mid}$ (resp. $\left.\alpha_{A \mid}\right)$ is the restriction of $\bar{\alpha}_{A}\left(\right.$ resp. $\left.\alpha_{A}\right)$ and $\phi(a \bigotimes b)=[a, b]=a b-b a$.

c) The induced Hom-Leibniz action of $\left(A, \alpha_{A}\right)$ on $\left(H H_{1}^{\alpha}(A), \alpha_{A \mid}\right)$ is trivial. Moreover, $i$ always preserves the actions and, if $\left(A, \alpha_{A}\right)$ satisfies the $\alpha$ identity condition (5.3), then $\phi$ also preserves the actions. 
Proof. a) By using formula (5.2) and Remark 5.2, one can readily check that the actions are well-defined Hom-Leibniz actions. It is also easy to see that these actions are compatible under condition (5.3). For example, we have

$$
\begin{aligned}
& { }^{\left.{ }^{a^{\prime}}(a \otimes b)\right)} a^{\prime \prime}={ }^{\left[a^{\prime}, a\right] \bowtie \alpha_{A}(b)} a^{\prime \prime}-{ }^{\left[a^{\prime}, b\right] \varangle \alpha_{A}(a)} a^{\prime \prime}=\left[\left[\left[a^{\prime}, a\right], \alpha_{A}(b)\right]-\left[\left[a^{\prime}, b\right], \alpha_{A}((a))\right], a^{\prime \prime}\right] \\
& \stackrel{\sqrt[2.1]{=}}{=}\left[\left[\alpha_{A}\left(a^{\prime}\right),[a, b]\right], a^{\prime \prime}\right] \stackrel{\sqrt[5.3]{=}}{=}\left[\left[a^{\prime},[a, b]\right], a^{\prime \prime}\right]=\left[a^{\prime a \bowtie b}, a^{\prime \prime}\right] \text {. }
\end{aligned}
$$

b) It is an immediate consequence of (5.1) and the definitions above.

c) Since ${ }^{a^{\prime}}(a \otimes b)=\alpha_{A}\left(a^{\prime}\right) \otimes[a, b]=0$ when $a \bigotimes b \in \operatorname{Ker}(\phi)$, we deduce that the action of $\left(A, \alpha_{A}\right)$ on $\left(H H_{1}^{\alpha}(A), \bar{\alpha}_{A \mid}\right)$ is trivial and, by this reason, $i$ preserves the actions of $\left(A, \alpha_{A}\right)$. Now, if $\left(A, \alpha_{A}\right)$ satisfies the $\alpha$-identity condition (5.3), then we have

$$
\begin{aligned}
& \phi\left(a^{\prime}(a \bigotimes b)\right)=\left[\alpha_{A}\left(a^{\prime}\right),[a, b]\right] \stackrel{5.30}{=}\left[a^{\prime},[a, b]\right]={ }^{a^{\prime}} \phi(a \bigotimes b), \\
& \phi\left((a \bigotimes b)^{a^{\prime}}\right)=\left[[a, b], \alpha_{A}\left(a^{\prime}\right)\right] \stackrel{5.3)}{=}\left[[a, b], a^{\prime}\right]=\phi(a \bigotimes b)^{a^{\prime}},
\end{aligned}
$$

for all $a^{\prime}, a, b \in A$, implying that $\phi$ also preserves the actions.

Remark 5.5. The statements in Proposition 5.4 when $\alpha_{A}=\mathrm{id}_{A}$ recover the corresponding results established in [9, Section 7] for Leibniz algebras.

In the following definition we introduce the Hom-version of the first Milnortype Hochschield homology of associative algebras (see e.g. [9]).

Definition 5.6. Let $\left(A, \alpha_{A}\right)$ be a Hom-associative algebra. The first Milnor-type Hochschield homology $H H_{1}^{M}\left(A, \alpha_{A}\right)$ is the quotient of the vector space $A \otimes A$ by the relations

$$
\begin{aligned}
a b \otimes \alpha_{A}(c)-\alpha_{A}(a) \otimes b c+c a \otimes \alpha_{A}(b) & =0, \\
\alpha_{A}(a) \otimes b c-\alpha_{A}(a) \otimes c b & =0, \\
a b \otimes \alpha_{A}(c)-b a \otimes \alpha_{A}(c) & =0,
\end{aligned}
$$

for any $a, b, c \in A$.

Theorem 5.7. Let $\left(A, \alpha_{A}\right)$ be a Hom-associative (non-commutative) algebra satisfying the $\alpha$-identity condition (5.3). Then there is an exact sequence of vector spaces

$$
\begin{aligned}
A * H H_{1}^{\alpha}(A) & \rightarrow \operatorname{Ker}\left(A * \mathbb{L}^{\alpha}(A) \rightarrow \mathbb{L}^{\alpha}(A)\right) \rightarrow \operatorname{Ker}(A *[A, A] \rightarrow[A, A]) \\
& \rightarrow H H_{1}^{\alpha}(A) \rightarrow H H_{1}^{M}\left(A, \alpha_{A}\right) \rightarrow[A, A] /[A,[A, A]] \rightarrow 0 .
\end{aligned}
$$


Proof. By using Proposition 5.4 and Proposition 3.8 we have the commutative diagram of Hom-Leibniz algebras (written without $\alpha$ endomorphisms)

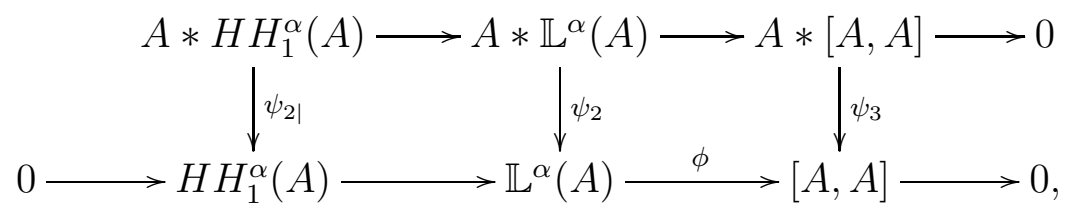

where $\psi$ homomorphisms are defined as in Proposition [3.10, $\psi_{2}(a *(x \otimes y))=$ ${ }^{a}(x \otimes y), \psi_{2}((x \otimes y) * a)=(x \otimes y)^{a}$ and $\psi_{3}(a *[x, y])=\left[\alpha_{A}(a),[x, y]\right], \bar{\psi}_{2}((x \otimes y) * a)=$ $\left[[x, y], \alpha_{A}(a)\right]$.

Thus $\operatorname{Coker}\left(\psi_{3}\right)=[A, A] /[A,[A, A]], \operatorname{Coker}\left(\psi_{2}\right)=H H_{1}^{M}\left(A, \alpha_{A}\right), \operatorname{Coker}\left(\psi_{2 \mid}\right)=$ $H H_{1}^{\alpha}(A)$ and $\operatorname{Ker}\left(\psi_{2 \mid}\right)=A * H H_{1}^{\alpha}(A)$. Then the assertion is a consequence of the Snake Lemma.

\section{Remark 5.8.}

a) Let $\left(A, \alpha_{A}\right)$ be a Hom-associative (non-commutative) algebra satisfying the $\alpha$-identity condition (5.3). If in addition $\alpha_{A}$ is an epimorphism, then the term $A * H H_{1}^{\alpha}(A)$ in the exact sequence of Theorem 5.7 can be replaced by $\left(A /[A, A] \otimes H H_{1}^{\alpha}(A)\right) \oplus\left(H H_{1}^{\alpha}(A) \otimes A /[A, A]\right)$ since they are isomorphic by Proposition 3.7. In Particular, if $\alpha_{A}=\mathrm{id}_{A}$, the exact sequence in Theorem 5.7 coincides with that of [9, Theorem 7.4].

b) From the exact sequence in Theorem 5.7 and Theorem 4.4 immediately follows that the vector spaces $H H_{1}^{\alpha}(A)$ and $H H_{1}^{M}\left(A, \alpha_{A}\right)$ are isomorphic when the Hom-associative algebra $\left(A, \alpha_{A}\right)$ is perfect as Hom-Leibniz algebra and $H L_{2}^{\alpha}(A)=0$.

c) $H H_{1}^{\alpha}(A)$ and $H H_{1}^{M}\left(A, \alpha_{A}\right)$ are also isomorphic for any commutative Homassociative algebra $\left(A, \alpha_{A}\right)$.

\section{Acknowledgements}

First and second authors were supported by Ministerio de Economía y Competitividad (Spain) (European FEDER support included), grant MTM2013-43687-P. The second author was supported by Xunta de Galicia, grants EM2013/016 and GRC2013-045 (European FEDER support included), and Shota Rustaveli National Science Foundation, grant FR/189/5-113/14.

\section{References}

[1] F. Ammar, S. Mabrouk and A. Makhlouf, Representations and cohomology of n-ary multiplicative Hom-Nambu-Lie algebras, J. Geom. Phys. 61 (10) (2011), 1898-1913. 
[2] S. Benayadi and A. Makhlouf, Hom-Lie algebras with symmetric invariant nondegenerate bilinear forms, J. Geom. Phys. 76 (2014), 38-60.

[3] J. M. Casas, M. A. Insua and N. Pacheco Rego, On universal central extensions of Hom-Leibniz algebras, J. Algebra Appl. 13 (8) (2014), 1450053 (22 pp.).

[4] J. M. Casas, E. Khmaladze and N. Pacheco Rego, A non-abelian tensor product of Hom-Lie algebras, Bull. Malaysian Math. Sci. Soc (2016), to appear.

[5] J. M. Casas and T. Van der Linden, Universal central extensions in semiabelian categories, Appl. Categor. Struct. 22 (1) (2014), 253-268.

[6] D. Chen and Y. Sheng, Hom-Lie 2-algebras, J. Algebra 376 (2013), 174-195.

[7] Y. S. Chen and Y. C. Su, (Co)homology and universal central extensions of Hom-Leibniz algebras, Acta Math. Sin. (Engl. Ser.) 27 (5) (2011), 813-830.

[8] G. Ellis, A non-abelian tensor product of Lie algebras, Glasgow Math. J. 33 (1991), 101-120.

[9] A. V. Gnedbaye, A non-abelian tensor product of Leibniz algebras, Ann. Inst. Fourier, Grenoble 49 (4) (1999), 1149-1177.

[10] J. T. Hartwing, D. Larson and S. D. Silvestrov, Deformations of Lie algebras using $\sigma$-derivations, J. Algebra 295 (2006), 314-361.

[11] N. Inassaridze, E. Khmaladze and M. Ladra, Non-abelian homology of Lie algebras, Glasgow Math. J. 46 (2004), 417-429.

[12] J.-L. Loday, Une version non commutative des algèbres de Lie: les algèbres de Leibniz, L'Enseignement Mathématique 39 (1993), 269-292.

[13] J.-L. Loday and T. Pirashvili, Universal enveloping algebras of Leibniz algebras and (co)homology, Math. Ann. 296 (1993), 139-158.

[14] A. Makhlouf and S. Silvestrov, Hom-algebra structures, J. Gen. Lie Theory Appl. 2 (2) (2008), 51-64.

[15] A. Makhlouf and S. Silvestrov, Hom-Lie admissible Hom-coalgebras and Hom-Hopf algebras, Generalized Lie theory in mathematics, physics and beyond, (Springer, Berlin 2009) 189-206.

[16] A. Makhlouf and S. Silvestrov, Notes on 1-parameter formal deformations of Hom-associative and Hom-Lie algebras, Forum Math. 22 (4) (2010), 715-739.

[17] Y. Sheng, Representations of Hom-Lie algebras, Algebr. Represent. Theory 15 (6) (2012), 1081-1098. 
[18] D. Yau, Enveloping algebras of Hom-Lie algebras, J. Gen. Lie Theory Appl. 2 (2) (2008), 95-108.

[19] D. Yau, Hom-algebras and homology, J. Lie Theory 19 (2) (2009), 409-421. 